\title{
Recent Advances in Acquiring Channel State Information in Cellular MIMO Systems
}

\author{
Gábor Fodor ${ }^{1,2}$, László Pap ${ }^{3}$ and Miklós Telek ${ }^{3,4}$
}

\begin{abstract}
In cellular multi-user multiple input multiple output (MU-MIMO) systems the quality of the available channel state information (CSI) has a large impact on the system performance. Specifically, reliable CSI at the transmitter is required to determine the appropriate modulation and coding scheme, transmit power and the precoder vector, while CSI at the receiver is needed to decode the received data symbols. Therefore, cellular MUMIMO systems employ predefined pilot sequences and configure associated time, frequency, code and power resources to facilitate the acquisition of high quality CSI for data transmission and reception. Although the trade-off between the resources used for pilot and user data transmission has been known for long, the nearoptimal configuration of the available system resources for pilot and data transmission is a topic of current research efforts. Indeed, since the fifth generation of cellular systems utilizes heterogeneous networks in which base stations are equipped with a large number of transmit and receive antennas, the appropriate configuration of pilot-data resources becomes a critical design aspect. In this article, we review recent advances in system design approaches that are designed for the acquisition of CSI and discuss some of the recent results that help to dimension the pilot and data resources specifically in cellular MU-MIMO systems.
\end{abstract}

Index Terms - Multi-antenna systems, channel state information, estimation techniques, receiver algorithms.

\section{INTRODUCTION}

In the uplink of cellular MU-MIMO systems, the base station (BS) typically acquires CSI of the uplink by means of uplink pilot or reference signals that are orthogonal in the code domain. Mobile stations (MSs) in long term evolution (LTE) systems, for example, use cyclically shifted Zadoff-Chu sequences to form demodulation reference signals allowing the BS to acquire CSI at the receiver (CSIR), which is necessary for uplink data reception [1]. By contrast, to acquire CSI at the transmitter (CSIT), BSs rely either on downlink pilots and quantized information fed back by MSs [2] or assume channel reciprocity [3]. It has been pointed out by several related works that in systems employing pilot aided channel estimation the number of pilot symbols and the pilot-to-data power ratio (PDPR) play a crucial role in optimizing the inherent tradeoff of sharing the available resources between pilot and data symbols [3]-[6].

The early work in [4] determined lower and upper bounds on the difference between the mutual information when the

G. Fodor is partially supported by the joint Ericsson-KTH project Machine Learning for Spectrum Sharing in Massive MIMO Networks (SPECS II). M. Telek is partially supported by the OTKA K-123914 and the TUDFO/51757/2019-ITM grants.

1,2 Ericsson Research, Stockholm, Sweden.E-mail: Gabor.Fodor@ericsson.com

${ }^{2}$ KTH Royal Institute of Technology, Stockholm, Sweden.E-mail: gaborf@kth.se

${ }^{3}$ Budapest University of Technology and Economics, Budapest, Hungary. E-mail: \{pap,telek\}@hit.bme.hu

${ }^{4}$ MTA-BME Information Systems Research Group, Budapest, Hungary.

DOI: 10.36244/ICJ.2019.3.2 receiver has an estimate of the CSI and when it has perfect knowledge of the channel. It also determined upper and lower bounds - as functions of the variance of the channel measurement error - on this difference. Subsequently, the results in [5] showed how pilot-based channel estimation affects the capacity of the fading channel, emphasizing that training imposes a substantial information-theoretic penalty, especially when the coherence interval $T$ (expressed in terms of the number of symbols available for pilot and data transmission) is only slightly larger than the number of transmit antennas $M$, or when the signal-to-noise ratio (SNR) is low. In these regimes, learning the entire channel is highly suboptimal. Conversely, if the SNR is high, and $T$ is much larger than $M$, training-based schemes can come very close to achieving capacity. Therefore, the power that should be spent on training and data transmission depends on the relation between $T$ and $M$. Specifically in MIMO orthogonal frequency division multiplexing (OFDM) systems that employ minimum mean squared error (MMSE) channel estimation, references [6] and [7] computed lower bounds. It was also shown that the optimal PDPR that maximizes this lower bound or minimizes the average symbol error rate can significantly increase the capacity compared with a system that uses a suboptimal PDPR setting. More recently, specifically for MU-MIMO systems, the trade-off between pilot and data symbols was analyzed in [8].

While the above references focused on a single cell system, a series of other works developed models for multi-cell MU-MIMO systems and proposed multi-cell pilot and/or data power control schemes that aim to maximize suitable systemwide utility functions [9]-[11]. In particular, the results in [9] and [10] indicate that in multi-cell MU-MIMO systems controlling the transmit power of both the pilot and data symbols can drastically improve the spectral and energy efficiency of the system. These papers assume the availability of a central control entity, which is hardly feasible in practice. Likewise, [10] demonstrates that multi-cell power control for the pilot and data symbols is necessary to maximize the system sum-rate, but it does not propose a decentralized algorithm that could be used for this purpose in practice. Therefore, suitable multi-cell schemes are actively researched by the academic and industrial communities.

In this direction, the work by [11] proposes a multi-cell game-theoretic approach for pilot contamination avoidance, although it does not consider the power control problem and that of setting the PDPR. The purpose of the present article is to survey recent advances and to point at some open problems in acquiring CSI in cellular MU-MIMO systems. Since understanding the inherent trade-offs of CSI acquisition is necessary to appreciate recent system design approaches and results, Section II provides a brief overview of the evolution of multi- 
antenna systems specifically in cellular networks. Next, Section III describes the two fundamental inherent trade-offs associated with CSI acquisition (related to the number of pilot symbols and the applied pilot power respectively). Section IV surveys recent papers related to CSI at the transmitter acquisition, that is of fundamental importance for downlink transmissions. Section $\mathrm{V}$ discusses advancements in CSI acquisition at the receiver, that is important for uplink reception and downlink transmission when reciprocity between the uplink and downlink channels holds. Reference signal design and channel estimation are discussed in Section VI. Next, Section VII provides an overview of recent papers that develop decentralized schemes that ease the burden on the base station by involving the mobile stations in the power control, resource allocation and channel estimation tasks. Finally, Section VIII discusses recent advances in mmWave systems, that are promising candidates for accommodating large scale MIMO systems and for taking advantage of underutilized spectrum resources. Section IX offers concluding remarks and provides an outlook on CSI acquisition in future cellular systems.

\section{The Evolution of Multi-Antenna Systems: From Single User to Massive Multi-USER Multiple InPUT Multiple Output Systems}

Conventional communication systems equipped with a single transmit antenna and a single receive antenna are called single input single output (SISO) communication systems (Figure 1, upper left). This intuitively clear terminology explicitly refers to a signal model that involves the convolution of the complex impulse response of the wireless channel (typically represented as a random variable $h$ ) and the single input $x$ to model the single output $y$ :

$$
y=h \star x+n,
$$

where $n$ is complex baseband additive white Gaussian noise (AWGN). The above equation is for a single realization of the complex single output $y$ [12].

The value of multiple antenna systems as a means to improve communications, including improving the overall system capacity and transmission reliability, was recognized in the early ages of wireless communications. Specifically, adaptive transmit or receive beamforming by means of employing multiple antennas either at the transmitter or the receiver roots back to classic papers that appeared in the 1960s and 1970s [13]-[15]. In particular, Widrow et al. described a least mean square (LMS) adaptive antenna array, which is a technique to adaptively determine the weights that are derived from the received signal to minimize the mean squared error (MSE) between the received signal and a reference (pilot) signal [13], [15]. Applebaum proposed a multiple antenna array structure that adaptively suppresses sidelobe energy when the desired signal's angle of arrival (AoA) is known, such as in a radar system.

Starting from the 1980's, there has been a renewed and increased interest in employing multiple antenna techniques in commercial systems, particularly mobile and cellular systems, where multipath and unintentional interference from simultaneously served users were the main concern [16]. However, it was

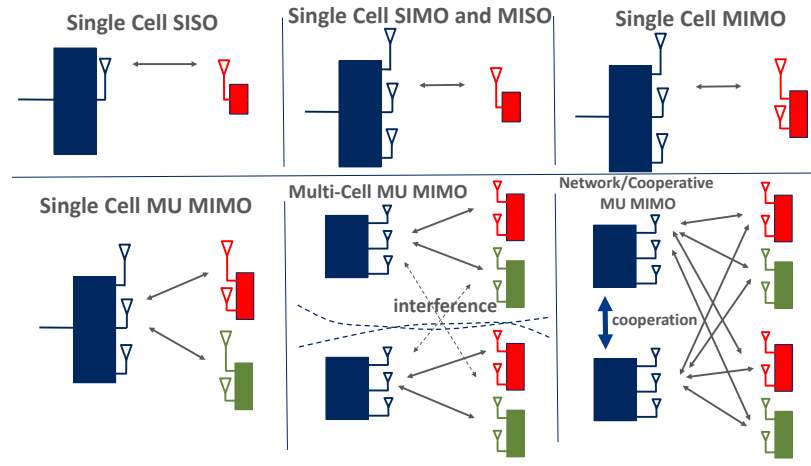

Figure 1. The evolution of multiple antenna systems from single cell single input single output transmissions to cooperative network multiple input multiple output transmissions.

not until the cost of digital signal processing was dramatically reduced and commercial wireless systems matured in the late 1990s that adaptive beamforming became commercially feasible, and large scale industrial interest has started to take off.

While traditional SISO systems exploit time- or frequencydomain processing and decoding of the transmitted and received data [17], [18], the use of additional antenna elements at the cellular BS or user equipment (UE) side opens up the extra spatial dimension to signal precoding and detection. Depending on the availability of multiple antennas at the transmitter and the receiver, such techniques are classified as Single Input Multiple Output (SIMO), Multiple Input Single Output (MISO) or MIMO (Figure 1, upper middle and upper right). Specifically, space-time and space-frequency processing methods in SIMO, MISO and MIMO systems make use of the spatial dimension with the aim of improving the link's performance in terms of error rate, data rate or spectral and energy efficiency [15].

In the context of cellular networks, for example, in the scenario of a multi-antenna enabled BS communicating with a single antenna UE, the uplink (UL) and downlink (DL) are referred to as SIMO and MISO respectively. When a multi-antenna terminal is involved, a full MIMO link may be obtained, although the term MIMO is sometimes also used in a collective sense including SIMO and MISO as special cases.

A MIMO system, in which the transmitter and receiver are equipped with $M$ and $N$ antennas respectively, is conveniently characterized by the multi-dimensional version of (1) as follows:

$$
\mathbf{y}=\underbrace{\mathbf{H}}_{N \times M} \underbrace{\mathbf{x}}_{M \times 1}+\underbrace{\mathbf{n}}_{N \times 1} \in \mathcal{C}^{N \times 1},
$$

where $\mathbf{x}$ and $\mathbf{y}$ represent the complex $M$ and $N$ dimensional input and output vectors of the MIMO system respectively and $\mathbf{n}$ is complex baseband AWGN vector.

While a point-to-point multiple-antenna link between a BS and a UE is referred to as Single-User Multiple Input Multiple Output (SU-MIMO), MU-MIMO features several UEs communicating simultaneously using the same frequency- and time-domain resources (Figure 1, lower left). By extension, considering a multi-cell system, neighboring BSs sharing their 
antennas and forming a virtual MIMO system to communicate with the same set of UEs in different cells are called cooperative multi-point (CoMP) or network MIMO transmission/reception (Figure 1, lower middle and lower right).

Multiple antenna techniques, as illustrated by Figure 1 offer (the combinations of) three advantages over traditional SISO systems:

- Diversity gain: The diversity gain corresponds to the mitigation of the effect of multipath fading, by means of transmitting and/or receiving over multiple wireless channels created by the multiple antennas on the transmit and/or receive sides of the communication link.

- Array gain: The array gain corresponds to a spatial version of the well-known matched-filter gain achieved by timedomain receivers.

- Spatial multiplexing gain: The spatial multiplexing gain refers to the ability to send multiple data streams in parallel and to separate them on the basis of their spatial signature. The spatial multiplexing gain is a particularly attractive gain of MIMO systems over SISO systems, because MIMO data stream multiplexing does not come at the cost of bandwidth expansion and can therefore yield drastic spectral efficiency gains.

As we shall see, the gains associated with multi-antenna systems strongly depend on the availability of CSI - the matrix $\mathbf{H}$ in (2) - at the transmitter and the receiver, which motivated the research and standardization communities to develop resource efficient techniques that enable the acquisition of CSIT and CSIR. Due to their great impact on the achievable gains, these acquisition techniques form an important part of MIMO systems, as discussed in more detail in the next section.

Due to the advances in digital signal processing, antenna theory and the commercial success of MIMO, and in particular, MU-MIMO systems, the research community has been investigating the characteristics of large scale antenna systems, in which the cellular BS is equipped with a great number of antennas. Indeed, evolving wireless standards are expected to support the deployment of several tens or even hundreds of transmit and receive antennas at infrastructure nodes and over ten transmit and receive antennas at commercial UEs. It is worth noting that in the asymptotic regime of such large scale or massive MIMO systems, it turns out that the lack of accurate CSI is the main cause of performance saturation, besides hardware impairments. Therefore, scalable and resource efficient CSI acquisition techniques have been and continues to be in the focus of the MIMO community ever since the large commercial deployments of such systems have started.

\section{Channel State Information ACQuisition AND} Transceiver Design: Challenges AND Trade-OFFs IN Multi-User Multiple InPut Multiple Output SYSTEMS

As noted, the spectral and energy-efficient operation of wireless systems in general, and multiple antenna systems in particular, relies on the acquisition of accurate CSIT and CSIR [19]. The main reasons for this are that (i) transmitters of modern wireless systems adapt the transmitted signal characteristics to the prevailing channel conditions and (ii) the effect of the channel on the transmitted signal must be estimated in order to recover the transmitted information. As long as the receiver accurately estimates how the channel modifies the transmitted signal, it can recover the signal from the impacts of the wireless channel. In practice, pilot signal-based data-aided techniques are used not only due to their superior performance in fast fading environments, but also due to their cost efficiency and inter-operability in commercial systems. Consequently, channel estimation methods have been studied extensively and a large number of schemes, including blind, data-aided, and decision-directed non-blind techniques, have been evaluated and proposed in the literature [20]-[22].

As the number of antennas at the BS and the simultaneously served users grow large, it is desirable to have pilot based schemes that are scalable in terms of the required pilot symbols and provide high quality CSI for UL data detection and DL precoding. To this end, MU-MIMO systems employing a large number of antennas typically rely on channel reciprocity and employ uplink pilots to acquire CSI at BSs. Although solutions for non-reciprocal systems (such as systems operating in frequency division duplexing (FDD) mode) are available [23], it is generally assumed that massive MIMO systems can advantageously operate in time division duplexing (TDD) mode exploiting channel reciprocity [3], [24].

Pilot reuse generally causes contamination of the channel estimates, which is known as pilot contamination (PC) or pilot pollution. As there are a large number of channels to be estimated in MU-MIMO and massive MIMO systems, accurate CSI acquisition scaling with the number of BS antennas becomes a significant challenge due to the potentially limited number of pilots available. Indeed, PC limits the performance gains of non-cooperative MU-MIMO systems [3], [25]. Specifically, $\mathrm{PC}$ is known to cause a saturation effect in the signal-tointerference-plus-noise ratio (SINR) as the number of BS antennas increases to a very large value. This is in contrast to the PC exempt scenario where the SINR increases almost linearly with the number of antennas [25]. It is therefore clear that the trade-offs associated with the resources used for pilot signals and those reserved for data transmission is a key design aspect of modern wireless communication systems.

Although pilot-based CSI acquisition is advantageous in fast fading environments, its inherent trade-offs must be taken into account when designing channel estimation techniques for various purposes. These purposes include demodulation, precoding or beamforming, spatial multiplexing and other channeldependent algorithms such as frequency selective scheduling or adaptive modulation and coding scheme (MCS) selection [6][8]. The inherent trade-offs between allocating resources to pilot and data symbols include the following, as illustrated in Figure 2:

- Increasing the power, time, or frequency resources to pilot signals improves the quality of the channel estimate, but leaves fewer resources for uplink or downlink data transmission [6]-[8].

- Constructing long pilot sequences (for example, employing orthogonal symbol sequences such as those based on the well-known Zadoff-Chu sequences in LTE systems) helps to avoid tight pilot reuse in multi-cell systems), helps to reduce or avoid inter-cell pilot interference. This is because 


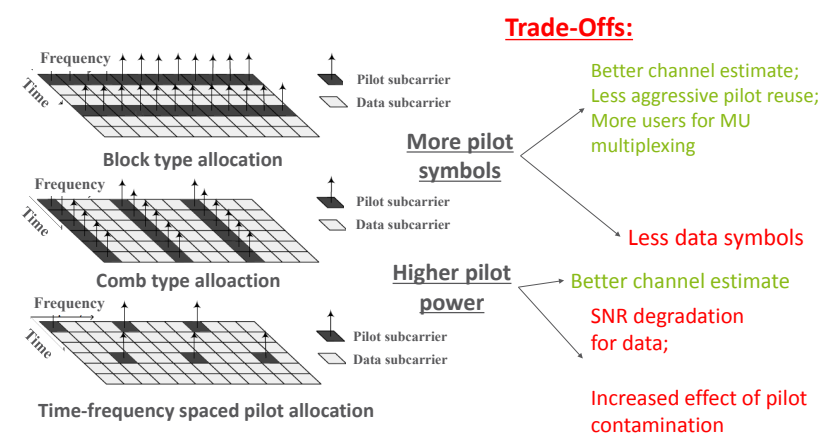

Figure 2. Trade-offs associated with channel estimation, reference (pilot) signal design in MU-MIMO systems

long pilot sequences enable to construct a great number of orthogonal sequences and, consequently, help to avoid pilot reuse in neighbor cells, and thereby address the root cause of PC. On the other hand, spending a greater number of symbols on pilots increases the pilot overhead and might violate the coherence bandwidth [8], [26].

- Specifically in MU-MIMO systems, increasing the number of orthogonal pilot sequences may increase the number of spatially multiplexed users at the expense of spending more symbols when creating the orthogonal sequences [6], [7].

In particular, increasing the pilot power increases the SNR of the received pilot signal, and thereby improves the quality of channel estimation in terms of the MSE of the channel estimate [27]. Unfortunately, increasing the pilot power may also lead to the SNR degradation of the data signals, and may exacerbate the PC problem in multi-cell scenarios [9]. In addition to these inherent trade-offs, the arrangement of the pilot symbols in the time, frequency, and spatial domains have been shown to have a significant impact on the performance of MU-MIMO and massive MIMO systems in practice, see for example [6], [7], [28].

\section{ReCEnt AdVAnCES in CSIT ACQuisition TECHNIQUES}

Recent research results and experiments with practical implementations have identified the key challenges that must be overcome in order to realize the potential benefits of massive MIMO [29]. One of the real-world challenges is given by the need of accurate CSI at the BS side. In principle, CSI may be obtained through transmitting orthogonal reference signals from each transmit antenna element, and then feeding back the observed spatial channel at the UE to the BS. This approach has the drawback that the reference signal overhead in terms of required CSI grows linearly with the number of transmit antennas. More specifically, CSIT at the transmitter in cellular systems employing FDD requires a feedback channel to the cellular BS, since reciprocity between the downlink and uplink channels cannot be assumed. When the number of antennas deployed at the BS is large, feedback-based CSIT acquisition is a challenge, because the number of pilot sequences as well as feeding back information about the entire vector channel

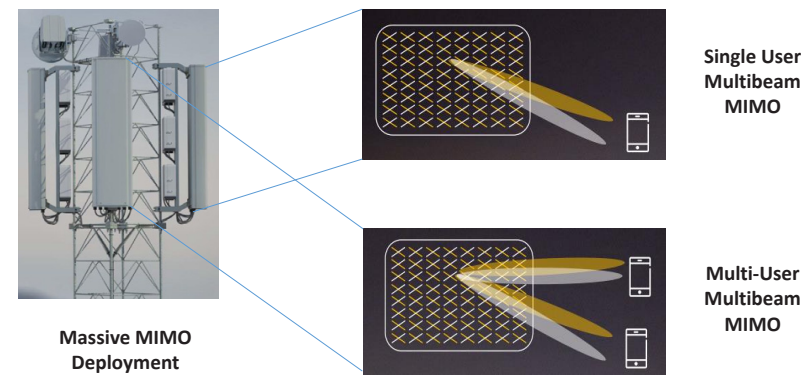

Figure 3. Massive MIMO deployments at the base station can support a large number of (up to several hundred) of possibly cross-polarized antenna elements. When high quality CSI is available at the BS, the system supports single and multi-user transmissions.

increases linearly with the number of antennas. For this reason, massive MU-MIMO systems are expected to be deployed in TDD systems, although valuable spectrum resources are allocated to FDD systems. Therefore, CSIT acquisition techniques that do not rely on channel reciprocity is of large interest by the research and standardization communities.

Indeed, one of the main technical goals of the 5th generation of cellular systems is to provide a system concept that supports 1000 times higher system spectral efficiency as compared with current LTE deployments but with a similar cost and energy dissipation per area as in today's cellular systems [30]. Historically, the 3rd Generation Partnership Project (3GPP) standard for the LTE has been designed with MU-MIMO as a goal to increase capacity. To this end, LTE has adopted various MU-MIMO technologies. Specifically, in LTE Release 8, the downlink transmission supports up to four antenna ports at the BS. There is an option for performing antenna switching with up to two transmit antennas. Furthermore, Release 10 (also known as LTE-Advanced or LTE-A) provides enhanced MIMO technologies. A new codebook and feedback design are implemented to support spatial multiplexing with up to eight independent spatial streams and enhanced MU-MIMO transmissions. The LTE Release 13 enables high-order MIMO systems with up to 64 antenna ports at the BS, which enables deployments in higher frequencies by supporting high-precision beamforming solution.

In a similar manner, massive or large MIMO systems are considered essential for meeting 5G capacity goals [24]. Massive MIMO systems generally have a large number of antennas at the BS consisting of 100 or more multiple antenna elements with associated large code books and scalable CSI acquisition techniques. An example of massive MIMO at the BS is shown in Figure 3. Clearly, these systems impose much more demanding requirements on CSI acquisition, precoding and receiver design in terms of scalability than the early release of LTE. Therefore, massive MIMO provides a suitable solution for substantially increasing the spectral efficiency and thereby the capacity for a given spectrum allocation. Massive MU-MIMO networks exploit the additional spatial degrees of freedom 
(DoF) to spatially multiplex the complex data symbols for several UEs scheduled on the same time-frequency resources in order to focus the radiated energy towards the intended receivers and to minimize the intracell and intercell interference [3], [24], [31].

As mentioned, the original massive MIMO downlink implementation is based on TDD operation, which allows to design near-optimal linear precoders, as CSIT for the downlink channels can be acquired through orthogonal uplink sounding exploiting channel reciprocity [32]. In contrast, in FDD operating mode, acquiring CSIT is more complex, since the channel estimation has to be carried out through downlink reference symbols (RSs) and subsequent uplink feedback. Therefore, in FDD systems, there exists a one-to-one correspondence between RSs and antenna elements. Consequently, in FDD systems, training and feedback overhead are often associated with unfeasibility in the massive MIMO regime, where a few resource elements (REs) are left for data transmission [33].

Nevertheless, operating in FDD remains appealing to mobile network operators for several reasons, including i) most radio bands below $6 \mathrm{GHz}$ are paired FDD bands, ii) the BSs have higher transmit power available for RSs than the UEs, and, as pointed out in [33], iii) overall deployment, operation and maintenance costs are reduced as fewer BSs are required in FDD networks. Moreover, as the number of UEs increases, longer orthogonal RSs are needed to avoid the so-called pilot contamination [32] - which increases power consumption at the UEs and the overall resource overhead.

To facilitate CSIT acquisition, in a way that scales well with the increasing number of antennas, the grid of beams (GoB) approach has been proposed in evolving $5 \mathrm{G}$ specifications [33], [34]. According to the GoB concept, a set of precoding vectors (that is a set of possible beams) is predefined, and the UEs see low-dimensional virtual (effective) channels instead of the actual ones, where the effective channels incorporate the precoding vectors. In particular, one orthogonal RS is allocated to each beam in the GoB codebook. Thus, estimating such effective channels reduces the overhead, as it becomes proportional to the codebook size (the number of possible beams) rather than to the number of antenna elements [35]. Unfortunately, the reduction in training overhead due to coarse granularity of the codebook, typically incurs some performance degradation [36], as the digital precoder for data transmission is based on a reduced channel representation, rather than a perantenna complex channel coefficient.

Another option for CSIT acquisition consists of designing the GoB with a large number of beams, and training a small subset of the available beams, which contains the dominant channel (multi-path) components of those beams [33], [37]. The number of such components depends on several factors, including the frequency band and the radio scattering environment, which are in general beyond the designer's control. Nevertheless, when multi-antenna UEs are deployed, statistical beamforming at the UE side can be exploited to let the UEs excite a suitable channel subspace, with the aim to further reduce the number of relevant components to be estimated [38], [39].

\section{RECENT ADVANCES IN CSIR AND RECIPROCITY-BASED CSIT ACQUISITION TECHNIQUES}

In fact, in $5 \mathrm{G}$ systems MSs are expected to have multiple antennas. Therefore, in 5G systems, the cost of utilizing reciprocity is that CSI acquisition requires array calibration in order to take the differences in the transmit/receive radio frequency (RF) chains of the different antenna elements at the BS and MS into account. In time varying channels, the delay between training and data transmission also represents an effect that should be further studied. For example, recent results indicate that channel prediction techniques can be used to mitigate this delay which would degrade the performance of massive MIMO systems [30].

In multi-cell and multi-tier cellular networks operating in TDD and utilizing channel reciprocity, reusing the pilot sequences leads to uplink pilot interference, often referred to as pilot contamination [3], [40]. In multi-cell MU-MIMO systems, the pilot-data resource allocation trade-off is intertwined with the management of intercell interference (contamination) both on the pilot and data signals and calls for rethinking the reference signal design of classical systems such as the 3GPP LTE system. Recent works provide valuable insights into the joint design of pilot and data channels in multi-cell massive MU-MIMO systems [41].

Some of the problems related to PDPR setting in MU-MIMO systems have been addressed by [8], [9], [26], [42]-[46]. Reference [8] considers a MU-MIMO scenario with time-division duplex operation, and a coherence interval of $T$ symbols spent for channel training, channel estimation, and precoder computation for DL transmission. The optimum number of pilot symbols is determined for maximizing the lower bound of the sum-throughput. However, receiver design and the PDPRsetting are out of the scope of that paper. The problem of joint power loading of data and pilot symbols for the purpose of maximizing sum spectral efficiency is addressed in [42], but the impact of PDPR setting at the MU-MIMO receiver is not considered. In contrast, the problem of optimal training period and update interval for maximizing the UL sum-rate is addressed in [44], whereas the receiver structure at the BS is not considered. Reference [26] considers single-user wireless fading channels, and optimizes the pilot overhead. That paper also identifies that the pilot overhead, as well as the spectral efficiency penalty, depends on the square root of the normalized Doppler frequency. More recently, uplink power control and the PDPR-setting problem in MU-MIMO systems have been addressed in references [9], [43], [47], [48], assuming practical (zero-forcing (ZF) and MMSE based) multi-antenna receiver structures. However, the papers mentioned above focus on centralized approaches, and may not scale well in multi-cell multi-user systems in practice. Scalable decentralized schemes with low complexity are appealing for PDPR setting in multicell MU-MIMO systems, and have been proposed in [28], [49][51].

\section{Reference Signal Design And ChanNel Estimation in Cellular MiMO Systems}

Due to the importance of CSI acquisition for data transmission and reception, it is natural, that designing reference (pilot) 
signals and employing accurate channel estimation techniques are of particular importance in cellular MIMO systems. The design of the uplink demodulation reference signals (DMRS) specifically in 3GPP Long Term Evolution Advanced (LTE-A) systems is described in [52]. In the LTE uplink, DMRS are used to facilitate channel estimation for the coherent demodulation of the physical uplink shared and control channels. The LTE DMRS occupies specific OFDM symbols within the uplink subframe according to the block type arrangement and supports a large number of user equipment utilizing cyclic extensions of the well known Zadoff-Chu sequences [53]. Because of its importance in practical systems, in this section we elaborate on the interplay between reference signal design and channel estimation in cellular MIMO systems, and refer to related works for further details.

To illustrate the design of reference signals in cellular MIMO systems, let us consider the uplink transmission of a multi-antenna single cell wireless system, in which users are scheduled on orthogonal frequency channels. In cellular MIMO systems, each MS employs an orthogonal pilot sequence, so that no interference between pilots within any given cell is present in the system. (Note that due to pilot reuse across neighbor cells, pilot contamination may still cause pilot interference.) This is a common practice in massive MU-MIMO systems in which a single MS may have a single antenna. The BS estimates the channel $\mathbf{h}$ (column vector of dimension $N_{r}$, where $N_{r}$ is the number of receive antennas at the BS) by either least squares (LS) or MMSE channel estimation to initialize an MMSE equalizer for uplink data reception. Since we employ orthogonal pilot sequences, the channel estimation process can be assumed independent for each MS within any given cell of the cellular system. Let us consider a time-frequency resource of $T$ time slots in the channel coherence time, and $F$ subcarriers in the coherence bandwidth, with a total number of symbols $\tau=F \cdot T$. We denote by $\tau_{p}$ the number of symbols allocated to pilots, and by $\tau_{d}$ the number of symbols allocated to data $\left(\tau_{p}+\tau_{d}=\tau\right)$. Moreover, we consider a transmission power level $P_{p}$ and $P$ for each pilot and data symbol, respectively. With this setup, we consider two pilot symbol allocation methods, namely block type and comb type, which we discuss in the following subsections. In practice, both of these schemes, and in fact, a combination of these are often used to construct uplink and downlink reference signals [52].

\section{A. Block Type Pilot Allocation}

The block type pilot arrangement consists of allocating one or more time slots for pilot transmission, by using all subcarriers in those time slots. This approach is a suitable strategy for slow time-varying channels. Given $T$ slots, a fraction of $T_{p}$ slots are allocated to the pilot and $T_{d}=T-T_{p}$ slots are allocated to the data symbols. Note that a maximum transmission power $P_{t o t}$ is allowed in each time slot, among all $F$ subcarriers. This power constraint is then identical for both the pilot $\left(P_{p}\right)$ and data power $(P)$, i.e.,

$$
F P_{p} \leq P_{\text {tot }} \quad F P \leq P_{t o t} .
$$

The power cannot be traded between pilot and data, but the energy budget can be distributed by tuning the number of time slots $T_{p}$ and $T_{d}$, i.e., $\tau_{p}=F T_{p}$ and $\tau_{d}=F T_{d}$.

\section{B. Comb Type Pilot Allocation}

In the comb type pilot arrangement a certain number of subcarriers are allocated to pilot symbols, continuously in time. This approach is a suitable strategy for non-frequency selective channels. Given $F$ subcarriers in the coherence bandwidth, a fraction of $F_{p}$ subcarriers are allocated to the pilot and $F_{d}=F-F_{p}$ subcarriers are allocated to the data symbols.

Each MS transmits at a constant power $P_{t o t}$, however, the transmission power can be distributed unequally in each subcarrier. In particular, if we consider a transmitted power $P_{p}$ for each pilot symbol and $P$ for each data symbol transmission, the following constraint is enforced:

$$
F_{p} P_{p}+F_{d} P=P_{t o t} .
$$

The total number of symbols for pilots is $\tau_{p}=T F_{p}$ and for data is $\tau_{d}=T F_{d}$. However, with comb type pilot arrangement, the trade-off between pilot and data signals includes the tradeoffs between the number of frequency channels and between the transmit power levels, which is an additional degree of freedom compared with the block type arrangement.

\section{Channel Estimation}

Let us consider a MS that transmits an orthogonal pilot sequence $\mathbf{s}=\left[s_{1}, \ldots, s_{\tau_{p}}\right]^{T}$, where each symbol is scaled as $\left|s_{i}\right|^{2}=1$, for $i=1, . ., \tau_{p}$, and ${ }^{T},{ }^{*}$, and ${ }^{H}$ denote the transpose, the conjugate and the conjugate transpose, respectively. Thus, the $N_{r} \times \tau_{p}$ matrix of the received pilot signal at the BS from the MS is:

$$
\mathbf{Y}^{p}=\alpha \sqrt{P_{p}} \mathbf{h} \mathbf{s}^{T}+\mathbf{N},
$$

where we assume that $\mathbf{h}$ is a circular symmetric complex normal distributed vector of r.v. with mean vector $\mathbf{0}$ and covariance matrix $\mathbf{C}$ (of size $N_{r}$ ), denoted as $\mathbf{h} \sim \mathcal{C N}(\mathbf{0}, \mathbf{C}$ ), $\alpha$ accounts for the propagation loss, $\mathbf{N} \in \mathbb{C}^{N_{r} \times \tau_{p}}$ is the spatially and temporally AWGN with element-wise variance $\sigma^{2}$.

In this paper, we consider two techniques, i.e., the LS and the MMSE channel estimation.

1) LS Estimation: Conventional LS estimation relies on correlating the received signal with the known pilot sequence. The BS estimates the channel based on assuming

$\hat{\mathbf{h}}_{L S}=\mathbf{h}+\tilde{\mathbf{h}}_{L S}=\frac{1}{\alpha \sqrt{P_{p}}} \mathbf{Y}^{p} \mathbf{s}^{*}\left(\mathbf{s}^{T} \mathbf{s}^{*}\right)^{-1}=\mathbf{h}+\frac{1}{\alpha \sqrt{P_{p}} \tau_{p}} \mathbf{N} \mathbf{s}^{*}$.

Note that $\mathbf{N ~ s}^{*}=\left[\sum_{i=1}^{\tau_{p}} s_{i}^{*} n_{i, 1}, \ldots, \sum_{i=1}^{\tau_{p}} s_{i}^{*} n_{i, N_{r}}\right]^{T}$, then $\mathbf{N} \mathbf{s}^{*} \sim \mathcal{C N}\left(\mathbf{0}, \tau_{p} \sigma^{2} \mathbf{I}_{N_{r}}\right)$.

By considering $\mathbf{h} \sim \mathcal{C N}(\mathbf{0}, \mathbf{C})$, it follows that the estimated channel $\hat{\mathbf{h}}_{L S}$ is a circular symmetric complex normal distributed vector $\hat{\mathbf{h}}_{L S} \sim \mathcal{C N}\left(\mathbf{0}, \mathbf{R}_{L S}\right)$, with

$$
\mathbf{R}_{L S}=\mathcal{E}\left\{\hat{\mathbf{h}}_{L S} \hat{\mathbf{h}}_{L S}^{H}\right\}=\mathbf{C}+\frac{\sigma^{2}}{\alpha^{2} P_{p} \tau_{p}} \mathbf{I}_{N_{r}} .
$$

The channel estimation error is defined as $\tilde{\mathbf{h}}_{L S}=\mathbf{h}-\hat{\mathbf{h}}_{L S}$, so that $\tilde{\mathbf{h}}_{L S} \sim \mathcal{C N}\left(\mathbf{0}, \mathbf{W}_{L S}\right)$ with

$$
\mathbf{W}_{L S}=\frac{\sigma^{2}}{\alpha^{2} P_{p} \tau_{p}} \mathbf{I}_{N_{r}}
$$


and the estimation MSE is derived as

$$
\varepsilon_{L S}=\mathcal{E}\left\{\left\|\tilde{\mathbf{h}}_{L S}\right\|_{F}^{2}\right\}=\operatorname{tr}\left\{\mathbf{W}_{L S}\right\}=\frac{N_{r} \sigma^{2}}{\alpha^{2} P_{p} \tau_{p}},
$$

where $\|\cdot\|_{F}^{2}$ is the Frobenius norm.

2) MMSE Estimation: We define a training matrix $\mathbf{S}=\mathbf{s} \otimes$ $\mathbf{I}_{N_{r}}$ (of size $\tau_{p} N_{r} \times N_{r}$ ), so that $\mathbf{S}^{H} \mathbf{S}=\tau_{p} \mathbf{I}_{N_{r}}$. The $\tau_{p} N_{r} \times 1$ vector of received signal can be conveniently rewritten as

$$
\tilde{\mathbf{Y}}^{p}=\alpha \sqrt{P_{p}} \mathbf{S h}+\tilde{\mathbf{N}},
$$

where $\tilde{\mathbf{Y}}^{p}, \tilde{\mathbf{N}} \in \mathbb{C}^{\tau_{p} N_{r} \times 1}$. The MMSE equalizer aims at minimizing the MSE between the estimate $\hat{\mathbf{h}}_{M M S E}=\mathbf{H} \tilde{\mathbf{Y}}^{p}$ and the actual channel realization $\mathbf{h}$. More precisely,

$$
\begin{aligned}
\mathbf{H} & =\arg \min _{\mathbf{H}} \mathcal{E}\left\{\left\|\mathbf{H} \tilde{\mathbf{Y}}^{p}-\mathbf{h}\right\|_{F}^{2}\right\} \\
& =\alpha \sqrt{P_{p}}\left(\sigma^{2} \mathbf{I}_{N_{r}}+\alpha^{2} P_{p} \mathbf{C S}^{H} \mathbf{S}\right)^{-1} \mathbf{C S}^{H} ; \quad \mathbf{H} \in \mathbb{C}^{N_{r} \times \tau_{p} N_{r}} .
\end{aligned}
$$

The MMSE estimate is then expressed as

$$
\begin{aligned}
\hat{\mathbf{h}}_{M M S E} & =\alpha \sqrt{P_{p}}\left(\sigma^{2} \mathbf{I}_{N_{r}}+\alpha^{2} P_{p} \tau_{p} \mathbf{C}\right)^{-1} \mathbf{C S}^{H}\left(\alpha \sqrt{P_{p}} \mathbf{S h}+\tilde{\mathbf{N}}\right) \\
& =\left(\frac{\sigma^{2}}{\alpha^{2} P_{p} \tau_{p}} \mathbf{I}_{N_{r}}+\mathbf{C}\right)^{-1} \mathbf{C}\left(\mathbf{h}+\frac{1}{\alpha \sqrt{P_{p}} \tau_{p}} \mathbf{S}^{H} \tilde{\mathbf{N}}\right) .
\end{aligned}
$$

Notice that $\mathbf{S}^{H} \mathbf{N} \sim \mathcal{C N}\left(\mathbf{0}, \tau_{p} \sigma^{2} \mathbf{I}_{N_{r}}\right)$, and therefore the estimated channel $\hat{\mathbf{h}}_{M M S E}$ is also a circular symmetric complex normal distributed vector $\hat{\mathbf{h}}_{M M S E} \sim \mathcal{C N}\left(\mathbf{0}, \mathbf{R}_{M M S E}\right)$, that is

$$
\hat{\mathbf{h}}_{M M S E}=\mathbf{h}+\tilde{\mathbf{h}}_{M M S E},
$$

and

$$
\mathbf{R}_{M M S E}=\mathbf{C}^{2}\left(\frac{\sigma^{2}}{\alpha^{2} P_{p} \tau_{p}} \mathbf{I}_{N_{r}}+\mathbf{C}\right)^{-1},
$$

where we considered $\mathbf{C}=\mathbf{C}^{H}$ and applied the commutativity of $\mathbf{C}$ and $\mathbf{I}_{N_{r}}$ to substitute

$$
\left(\frac{\sigma^{2}}{\alpha^{2} P_{p} \tau_{p}} \mathbf{I}_{N_{r}}+\mathbf{C}\right)^{-1} \mathbf{C}=\mathbf{C}\left(\frac{\sigma^{2}}{\alpha^{2} P_{p} \tau_{p}} \mathbf{I}_{N_{r}}+\mathbf{C}\right)^{-1}
$$

The channel estimation error is $\tilde{\mathbf{h}}_{M M S E}=\mathbf{h}-\hat{\mathbf{h}}_{M M S E}$ so that $\tilde{\mathbf{h}}_{M M S E} \sim \mathcal{C N}\left(\mathbf{0}, \mathbf{W}_{M M S E}\right)$ with

$$
\mathbf{W}_{M M S E}=\mathbf{C}\left(\mathbf{I}_{N_{r}}+\frac{\alpha^{2} P_{p} \tau_{p}}{\sigma^{2}} \mathbf{C}\right)^{-1}
$$

and the estimation MSE simply follows as

$$
\varepsilon_{M M S E}=\operatorname{tr}\left\{\mathbf{C}\left(\mathbf{I}_{N_{r}}+\frac{\alpha^{2} P_{p} \tau_{p}}{\sigma^{2}} \mathbf{C}\right)^{-1}\right\} .
$$

Notice that for both LS and MMSE channel estimation, the estimation MSE is a monotonically decreasing function of the pilot energy per antenna $P_{p} \tau_{p}$. Building on the characteristics of LS and MMSE channel estimation techniques, several research contributions characterize the receiver and the uplink signal MSE and spectral efficiency based on $\tilde{\mathbf{h}}$, which is computed for LS $\left(\hat{\mathbf{h}}_{L S}\right)$ and MMSE estimation ( $\left.\hat{\mathbf{h}}_{M M S E}\right)$ [54], [55].

\section{DECENTRALIZED APPROACHES TO CSI ACQUISITION}

As the number of antennas and the number of simultaneously served users by a single BS increase, decentralized algorithms for MU-MIMO systems become important, because they help to reduce the required processing power at a single entity such as the cellular base station. Therefore, there is an increasing interest in decentralized optimization schemes for MU-MIMO systems, see for example [28], [49]-[51]. These papers either assume the availability of perfect CSI, or incorporate CSI errors, but do not address the joint optimization of setting the pilot and the data power. A different line of work proposed a game theoretic approach for decentralized power control and resource allocation in multi-user (MU) systems in which some form of "performance coupling" [56] exists among the users, as the increase of one user's performance degrades the performance of others, e.g., [57] and [58]. These references suggest that game theoretic approaches in MU systems are appealing, because they naturally admit decentralized algorithms that can be easily deployed by both network nodes and MSs. It is, however, unclear whether a game theoretic treatment could be used for designing low complexity decentralized algorithms for setting the PDPR in MU cellular systems.

Due to its central role in the performance of MIMO systems, many recent work investigated the performance impact of the PDPR and proposed optimal or near-optimal schemes for setting the PDPR. The MU-MIMO scenario is analyzed in [8], in which the coherence interval of $T$ symbols is spent for channel training, channel estimation, and precoder computation for DL transmission. Specifically, the optimum number of terminals in terms of the DL spectral efficiency is determined for a given coherence interval, number of base station antennas, and SINR. There is no receiver design involved and the pilot-to-data power trade-off is out of the scope of the considered optimization problem. The joint power loading of data and pilot symbols for the purpose of acquiring CSIT for precoding is considered in [42], but the impact of setting the PDPR at the MU-MIMO receiver is not considered. In contrast, the UL sum-rate maximization problem by tuning the training period in a frequencyflat fading channel is considered in [44], without modeling the receiver structure at the BS. Reference [45] proposes a pilot design that maximizes the spectral efficiency of high mobility wireless communication systems that use pilot-assisted MMSE channel estimation. That work does not explicitly model the impact of CSI errors on MU-MIMO receivers, such as an MMSE receiver. Reference [26] investigated the optimization of the pilot overhead for single-user wireless fading channels, and the dependencies of this pilot overhead on various system parameters of interest (e.g. fading rate, SNR) were quantified. By finding an expansion of the spectral efficiency for the overhead optimization in terms of the fading rate around the perfect-CSI point, the square root dependence of both the overhead and the spectral efficiency penalty was clearly identified.

Another set of related papers develop decentralized optimization schemes for MIMO systems, either assuming the availability of perfect channel state information, or incorporating channel state information errors, but do not address the joint optimization of pilot and data power setting, see for example [28], [49]-[51]. 
Also, a number of recent work proposed a game theoretic approach for power control and resource allocation in $\mathrm{MU}$ systems in which performance coupling exists among the users, as the increase of one user's performance degrades the performance of others [56], [59], [57], [58], [60], [61] and [62]. The MU power control problem for the Gaussian frequencyflat relay channel is modelled as a Gaussian interference relay game (GIRG) in [56]. In the GIRG, instead of allocating the power budget across the set of sub-channels, each player aims to decide the optimal power control strategy across a set of hops. For cooperative cognitive radio networks, a coalitional game theoretic approach is proposed in [59]. The coalitional game model captures a cooperative secondary spectrum access scenario, and involves primary and secondary spectrum users such that the secondary users can act as cooperative relays for the primary users. A non-cooperative feedback-rate control game with pricing is considered in [57], as a model of the downlink transmission of a closed-loop wireless network, in which a multi-antenna BS utilizes CSI feedback to properly set linear precoders to communicate with multiple users. Reference [58] proposes a distributed power splitting scheme for simultaneous wireless information and power transfer in relay interference channels, where multiple source-destination pairs communicate through energy harvesting relays. The authors in [60] model power control as a non-cooperative game between transmitter-receiver pairs and show the existence of equilibria using quasi-variational inequality theory. Reference [61] formulates the problem of downlink power control of small cell base stations under a total power constraint as a generalized Nash equilibrium problem and proves the existence of equilibria. The authors in [62] consider a game theoretical formulation of the improper graph multi-coloring problem as a model of resource allocation between transmitter-receiver pairs, prove the existence of equilibria and provide polynomial complexity algorithms for computing equilibria.

A powerful game theoretic framework for the noncooperative maximization of mutual information assuming Gaussian interference channels in MU-MIMO systems is developed in [63]. As it is pointed out by [63], the main difficulty in the MIMO case as compared with SISO systems is that the optimal transmit directions of each MS change with the strategies of the other users, as opposed to the SISO case, where only the power allocation depends on the strategies of the other MSs. However, this framework assumes the availability of perfect CSI and does not address the trade-off between data transmission and channel estimation. In contrast, the work reported in [64] develops a game theoretic approach to maximizing the own information rates subject to transmit power and robust interference constraints allowing for non-perfect CSI availability at the transmitters and receivers specifically in a cognitive radio environment. However, the aspect of tuning the pilot and data power levels subject to a sum power constraint is not considered. For the DL, reference [65] assumes perfect CSIT at the BS and proposes a partially asynchronous distributed algorithm based on a non-cooperative game to find the DL precoders in MU-MIMO systems.

The MIMO scheme proposed in [46] considers the problem of joint pilot and data power control for the MU-MIMO UL. However, the model of [46] uses a receiver that minimizes the
MSE of the estimated data symbols only when perfect CSI is available. As it has been shown in our previous work [47], the performance of this naive receiver can be significantly improved by regularizing the receiver with respect to the statistics of the CSI estimation errors.

Recognizing the importance of scalable CSI acquisition approaches, in our recent work we propose a game theoretic approach to setting the PDPR in the UL of MU-MIMO systems and proposed decentralized algorithms that can be implemented in practice and converge to a unique Nash equilibrium [66], [67]. The contribution of those papers is a decentralized MU (pilot and data) power allocation algorithm, which we refer to as Best PDPR Algorithm (BPA). The numerical results obtained by testing BPA in a MU-MIMO system employing an increasing number of receive antennas yield several unique insights. Our results showed that BPA performs close to the globally optimal solution, which minimizes the sum of MSEs in MU-MIMO systems, and outperforms the traditional pilot power setting scheme that uses a fixed, predefined PDPR [66].

\section{TOWARDS MMWAVE FREQUENCY BANDS}

As discussed in this paper, Large-scale MIMO (LS-MIMO) systems involving an order of magnitude greater number of antenna elements than in the early releases of wireless standards are key enablers of next generation cellular systems and providing mobile broadband services [32]. Theoretically, a fully digital LS-MIMO beamforming architecture employing a large number of digital transmit and receiver chains, combined with resource efficient CSI acquisition techniques, near-optimal receiver design and employing decentralized power control schemes can yield near-optimal performance in terms of energy and spectral efficiency [68].

However, deploying LS-MIMO systems in traditional cellular frequency bands is also problematic due to the large physical size of the antenna arrays and related environmental concerns of the general public. Therefore, higher frequency bands, including the millimeter-wave (mmWave) bands have recently emerged as an appealing alternative for the commercial deployment of LS-MIMO systems [69]. Indeed, in mmWave bands, the physical array size can be greatly reduced, and, as an additional advantage, vast amount of unused spectrum can be utilized for attractive and bandwidth-demanding services [70], [71].

Deploying a large number of antennas with the associated fully digital beamforming architecture incurs high cost and increased power consumption, due to the excessive demand for a large number of transceiver chains. Therefore, LS-MIMO systems with hybrid analog and digital beamforming for mmWave deployment have attracted much attention from the research and engineering communities, and a great number of promising hybrid architectures and associated technologies such as training sequence and codebook designs have been proposed and tested in practice [72]-[76]. The results of the marriage of LS-MIMO and hybrid beamforming include significant gains in terms of spectral and energy efficiency, and a cost-efficient technology for accessing large amount of unused spectrum [68], [75], [77].

Specifically, in the framework of mmWave communications, [78]-[80] have studied the effect of hardware impairments on the performance of MIMO systems. The results of [78] 
show that single-carrier frequency domain equalization is more robust against impairments from nonlinear power amplifiers than OFDM in typical mmWave system configurations. On the other hand, the results reported in [79] show a slight bit error rate performance advantage of OFDM over single-carrier frequency domain equalization under nonlinear RF distortions, and suggest that subcarrier spacing is a crucial parameter in mmWave massive MIMO systems.

\section{CONCLUDING REMARKS}

In this survey paper, we discussed recent advances in the field of CSI acquisition and managing the inherent the tradeoff between using time, frequency and power resources for CSI acquisition and transmitting data symbols. Managing this tradeoff has a large impact on the achievable spectral efficiency in cellular systems, in which the number of transmit and receive antennas grows large. We made the point that the joint allocation of frequency, time and power resources is subject to constraints that depend on the specific pilot pattern, such as the pattern used by the block or comb type arrangements of pilot (reference) symbols.

Recent research results suggest that with a large number of antennas, exploiting the engineering freedom of tuning both the number of pilot symbols and the pilot transmit power levels become increasingly important, especially if the relatively simple LS estimator is used at the base station. Also, the gain of using MMSE estimation (preferably with optimized pilot power allocation) increases over LS estimation. Interestingly, the optimal amount of pilot and data resources is different when using MMSE and LS estimators and the gain in terms of spectral efficiency when optimizing both the number of pilot symbols and the transmit power levels increases as the number of antennas increases. In practical systems, decentralized schemes in which mobile stations and base stations participate in finding near optimal resource allocations become important, because decentralized schemes scale well with the number of served users and the number of antennas. A new field of research deals with resource allocation and CSI acquisition in millimeter-wave systems.

\section{REFERENCES}

[1] Stefania Sesia, Issam Toufik, and Matthew Baker. LTE - The UMTS Long Term Evolution: From Theory to Practice. WILEY, 2nd edition, 2011. ISBN-10: 0470660252.

[2] D. Gesbert, M. Kountouris, R. W. Heath Jr., C.-B. Chae, and T. Salzer. Shifting the MIMO paradigm: From single-user to multiuser communications. IEEE Signal Processing Magazine, 24(5):36-46, October 2007, DOI: 10.1109/MSP.2007.904815.

[3] T. Marzetta. Noncooperative cellular wireless with unlimited numbers of base station antennas. IEEE Trans. Wireless Comm., 9(11):3590-3600, 2010, DOI: 10.1109/TWC.2010.092810.091092.

[4] M. Médard. The effect upon channel capacity in wireless communications of perfect and imperfect knowledge of the channel. IEEE Trans. on Information Theory, 46(3):933-946, May 2000, DOI: 10.1109/18.841172.

[5] B. Hassibi and B. M. Hochwald. How much training is needed in multipleantenna wireless links? IEEE Trans. on Information Theory, 49(4):951963, April 2003, DOI: 10.1109/TIT.2003.809594.

[6] T. Kim and J. G. Andrews. Optimal pilot-to-data power ratio for MIMOOFDM. In IEEE Globecom, pages 1481-1485, St. Louis, MO, USA, Dec. 2005. DOI: 10.1109/GLOCOM.2005.1577897.
[7] T. Kim and J. G. Andrews. Balancing pilot and data power for adaptive MIMO-OFDM systems. In IEEE Globecom, San Francisco, CA, USA, Dec 2006. DOI: 10.1109/GLOCOM.2006.47.

[8] T. Marzetta. How much training is needed for multiuser MIMO? IEEE Asilomar Conference on Signals, Systems and Computers (ACSSC), pages 359-363, June 2006, DOI: 10.1109/ACSSC.2006.354768.

[9] K. Guo, Y. Guo, G. Fodor, and G. Ascheid. Uplink power control with MMSE receiver in multi-cell MU-Massive-MIMO systems. In Proc. of IEEE International Conference on Communications (ICC), pages 51845190, Jun. 2014, DOI: 10.1109/ICC.2014.6884144.

[10] X. Li, E. Björnsson, E. G. Larsson, S. Zhou, and J. Wang. Massive MIMO with multi-cell MMSE processing: Exploiting all pilots for interference suppression. arXiv:1505.03682v2 [cs.IT], May 2015.

[11] H. Ahmadi, A. Farhang, N. Marchetti, and A. MacKenzie. A game theoretic approach for pilot contamination avoidance in massive MIMO. IEEE Wireless Communications Letters, 5(1):12-15, February 2016, DOI: 10.1109/LWC.2015.2487261.

[12] Iana Siomina, Anders Furuskär, and Gabor Fodor. A mathematical framework for statistical QoS and capacity studies in OFDM networks. In Personal, Indoor and Mobile Radio Communications (PIMRC), pages 2772 - 2776, 10 2009, DOI: 10.1109/PIMRC.2009.5450287.

[13] B. Widrow, P. E. Mantey, L. J. Griffiths, and B. B. Goode. Adaptive antenna systems. Proceedings of the IEEE, 55(12):2143-2159, December 1967, DOI: 10.1109/PROC.1967.6092.

[14] S. R. Applebaum. Adaptive arrays. IEEE Transactions on Antennas and Propagation, 24(5):585-598, September 1976, DOI: 10.1109/TAP.1976.1141417

[15] J. Winters. Space-Time Wireless Systems - From Array Processing to MIMO Communications. Number ISBN:9780511279942. Cambridge University Press, 2006.

[16] J. H. Winters. Optimum combining in digital mobile radio with cochannel interference. IEEE Journal on Selected Areas in Communications, 2(4):528-539, April 1984, DOI: 10.1109/T-VT.1984.24001.

[17] Ádám Knapp and László Pap. General performance analysis of binary fading channels with measurement based feedback channel equalization. Infocommunications Journal, 1:1-9, 2014.

[18] Ádám Knapp and László Pap. Statistical based optimization of number of pilot signals in LTE/LTE-A for higher capacity. In IEEE EUROCON 2015 - International Conference on Computer as a Tool (EUROCON), pages 1-5, Sep. 2015, DOI: 10.1109/EUROCON.2015.7313768.

[19] A. Goldsmith, S. A. Jafar, N. Jindal, and S. Vishwanath. Capacity limits of MIMO channels. IEEE Journal on Selected Areas of Communications, 21(5):684-702, June 2003, DOI: 10.1109/JSAC.2003.810294

[20] M. K. Ozdemir and H. Arslan. Channel estimation for wireless OFDM systems. IEEE Communications Surveys and Tutorials, 9(2):18-48, 2007, DOI: 10.1109/COMST.2007.382406.

[21] S. Coleri, M. Ergen, A. Puri, and A. Bahai. Channel estimation techniques based on pilot arrangement in OFDM systems. IEEE Transactions on Broadcasting, 48(3):223-229, September 2002,

DOI: 10.1109/TBC.2002.804034.

[22] Y-H Nam, Y. Akimoto, Y. Kim, M i. Lee, K. Bhattad, and A. Ekpenyong Evolution of reference signals for LTE-advanced systems. IEEE Communications Magazine, 5(2):132-138, February 2012 DOI: 10.1109/MCOM.2012.6146492.

[23] J. Choi, T. Kim, D. J. Love, and J-Y. Seol. Exploiting the preferred domain of FDD massive MIMO systems with uniform planar arrays. In IEEE International Conference on Communications, pages 3068-3073, London, UK, June 2015. DOI: 10.1109/ICC.2015.7248530.

[24] F. Rusek, D. Persson, B. K.Lau, E. G. Larsson, T. L. Marzetta, O. Edfors, and F. Tufvesson. Scaling Up MIMO - Opportunities and Challenges with Very Large Arrays. IEEE Signal Processing Magazine, 30(1):40 - 60, January 2013, DOI: 10.1109/MSP.2011.2178495.

[25] B. Gopalakrishnan and N. Jindal. An analysis of pilot contamination on multiuser MIMO cellular systems with many antennas. In International Workshop on Signal Processing Advances in Wireless Communications, pages 381-385, San Francisco, June 2011. DOI: 10.1109/SPAWC.2011.5990435.

[26] N. Jindal and A. Lozano. A unified treatment of optimum pilot overhear in multipath fading channels. IEEE Trans. on Communications, 58(10):2939_ 2948, October 2010, DOI: 10.1109/TCOMM.2010.083110.090696

[27] G. Fodor and M. Telek. On the pilot-data power trade off in single input multiple output systems. European Wireless '14, Barcelona, Spain(ISBN 978-3-8007-3621-8), May 2014.

[28] H. Yin, D. Gesbert, M. Filippou, and Y. Liu. A Coordinated Approach to Channel Estimation in Large-Scale Multiple-Antenna Systems. IEEE Journal on Selected Areas in Communications, 31(2):264-273, February 2013, DOI: 10.1109/JSAC.2013.130214. 
[29] L. Lu, G. Li, A. Swindlehurst, A. Ashikhmin, and R. Zhang. An overview of massive MIMO: Benefits and challenges. IEEE Journal 9 of Selected Topics in Signal Processing, 8(5):742-758, October 2014, DOI: 10.1109/JSTSP.2014.2317671.

[30] Nandana Rajatheva, Satoshi Suyama, Wolfgang Zirwas, Lars Thiele, Gabor Fodor, Antti Tölli, Elisabeth Carvalho, and Jesper Hemming Sörensen. $5 G$ Mobile and Wireless Communications Technology. Cambridge University Press, 2016.

[31] J. Hoydis, S. T. Brink, and M. Debbah. Massive MIMO: How many antennas do we need? 49th Annual Allerton Conference on Communication, Control and Computing, pages 545-550, 2011, DOI: 10.1109/Allerton.2011.6120214.

[32] E. G. Larsson, O. Edfors, F. Tufvesson, and T. L. Marzetta. Massive MIMO for next generation wireless systems. IEEE Commun. Mag., Feb. 2014, DOI: 10.1109/MCOM.2014.6736761.

[33] W. Zirwas, M. B. Amin, and M. Sternad. Coded CSI reference signals for $5 \mathrm{G}$ - exploiting sparsity of FDD massive MIMO radio channels. In Proc. IEEE WSA, number ISBN:978-3-8007-4177-9, Mar. 2016.

[34] 3GPP. NR; physical layer procedures for data - Rel. 15, TS 38.214. Dec. 2018.

[35] F. Maschietti, G. Fodor, D. Gesbert, and P. de Kerret. Coordinated beam selection for training overhead reduction in FDD massive MIMO. Proc. IEEE International Symposium on Wireless Comm. Systems, ISWCS, Aug. 2019.

[36] J. Flordelis, F. Rusek, F. Tufvesson, E. G. Larsson, and O. Edfors. Massive MIMO performance - TDD vs FDD: What do measurements say? IEEE Trans. Wireless Commun., Apr. 2018, DOI: 10.1109/TWC.2018.2790912.

[37] F. Maschietti, D Gesbert, P de Kerret, and H. Wymeersch Robus location-aided beam alignment in millimeter wave massive MIMO. Proc. IEEE GLOBECOM, Dec. 2017, DOI: 10.1109/GLOCOM.2017.8254901.

[38] N. N. Moghadam, H. Shokri-Ghadikolaei, G. Fodor, M. Bengtsson, and C. Fischione. Pilot precoding and combining in multiuser MIMO networks IEEE J.Sel.Areas Commun., Jul. 2017, DOI: 10.1109/JSAC.2017.2699398.

[39] P. Mursia, I. Atzeni, D. Gesbert, and L. Cottatellucci. Covariance shaping for massive MIMO systems. In Proc. IEEE GLOBECOM, Dec. 2018 , DOI: 10.1109/GLOCOM.2018.8647861.

[40] J. Jose,A.Ashikhmin, T. Marzetta, and S. Vishwanath. Pilot contamination and precoding in multi-cell TDD systems. IEEE Transactions on Wireless Communications, 10(8):2640-2651, August 2011, DOI: 10.1109/TWC.2011.060711.101155.

[41] M. Kurras, L. Thiele, and G. Caire. Interference mitigation and multiuser multiplexing with beam-steering antennas. In 19th International ITG Workshop on Smart Antennas, number ISBN:978-3-8007-3662-1, pages 1-5, Ilmenau, Germany, March 2015.

[42] C. P. Sukumar and R. MerR. Merched. Eltawil. Joint power loading of data and pilots in OFDM using imperfect channel state information at the transmitter. In IEEE Global Communications Conference, pages 1-5, Nov. 2008, DOI: 10.1109/GLOCOM.2008.ECP.872.

[43] K. Min, M. Jung, T. Kim, Y. Kim, J. Lee, and S. Choi. Pilot power ratio for uplink sum-rate maximization in zero-forcing based MUMIMO systems with large number of antennas. In IEEE Vehicular Technology Conference (VTC-Fall), pages 1-5, 2-5 September 2013 , DOI: 10.1109/VTCFall.2013.6692364.

[44] Kien T. Truong, A. Lozano, and R. W. Heath Jr. Optimal training in continuous block-fading massive MIMO systems. 20th European Wireless, Barcelona, Spain, May 2014.

[45] N. Sun and J. Wu. Maximizing spectral efficiency for high mobility systems with imperfect channel state information. IEEE Trans. Wireless Communication, 13(3):1462-1470, March 2014, DOI: 10.1109/TWC.2014.012314.130772.

[46] K. Guo, Y. Guo, and G. Ascheid. Energy-efficient uplink power allocation in multi-cell MU-Massive-MIMO systems. In Proc. of European Wireless, number ISBN:978-3-8007-3976-9, pages 1-5, Budapest, Hungary, May 2015.

[47] G. Fodor, P. Di Marco, and M. Telek. On the impact of antenna correlation and CSI errors on the pilot-to-data power ratio. IEEE Transactions on Communications, 64(6):2622 - 2633, April 2016, DOI: 10.1109/TCOMM.2016.2549536.

[48] M. Sajadieh, A. Esswie, A. Fouda, H. Shirani-Mehr, and D. Chatterjee. Progressive channel state information for advanced multi-user mimo in next generation cellular systems. In 2016 IEEE Wireless Communications and Networking Conference, pages 1-6, April 2016, DOI: 10.1109/WCNC.2016.7564830.

[49] M. Ding and S. D. Blostein. Relation between joint optimizations for multiuser MIMO uplink and downlink with imperfect CSI. In IEEE International Conference on Acoustics, Speech and Signal Processing (ICASSP), pages 3149 - 3152, Las vegas, NV, USA, March 31-April 4 2008. DOI: 10.1109/ICASSP.2008.4518318.
[50] J. Kron, D. Persson, M. Skoglund, and E. G. Larsson. Closed-form SumMSE minimization for the two-user gaussian MIMO broadcast channel. IEEE Communications Letters, 15(9):950-952, Sep. 2011,

DOI: 10.1109/LCOMM.2011.070711.110571.

[51] J. Wang, M. Bengtsson, B. Ottersten, and D. Palomar. Robust MIMO precoding for several classes of channel uncertainty. IEEE Trans. Signal Processing, 61(12):3056-3070, April 2013, DOI: 10.1109/TSP.2013.2258016.

[52] B. Furht and S. A. Ahson. Long Term Evolution: 3GPP LTE Radio and Cellular Technology. Auerbach Publications, ISBN-10: 1420072102, April 2009.

[53] X. Hou, Z.Zhang, and H. Kayama. DMRS Design and Channel Estimation for LTE-Advanced MIMO Uplink. 70th IEEE Vehicular Technology Conference (Fall), pages 1-5,2009,DOI: 10.1109/VETECF.2009.5378829.

[54] G. Fodor, P. Di Marco, and M. Telek. Performance analysis of block and comb type channel estimation for massive MIMO systems. In First International Conference on $5 G$ for Ubiquitous Connectivity (5GU), pages 62-69, Nov. 2014, DOI: 10.4108/icst.5gu.2014.258076.

[55] G. Fodor, P. D. Marco, and M. Telek. On the impact of antenna correlation on the pilot-data balance in multiple antenna systems. In IEEE International Conference on Communications (ICC), pages 2590-2596, London, UK, Jun. 2015. DOI: 10.1109/ICC.2015.7248715.

[56] Y.Shi,J.Wang, K. B.Letaief, and R. K. Mallik.A game-theoretic approach for distributed power control in interference relay channels. IEEE Trans. On Wireless Communications, 8(6):3151-3161, 2009, DOI: 10.1109/TWC.2009.080831.

[57] L. Song, Z. Han, Z. Zhang, and B. Jiao. Non-cooperative feedback-rate control game for channel state information in wireless networks. IEEE Journal on Selected Areas in Communications, 30(1):188-197, 2012, DOI: 10.1109/JSAC.2012.120117.

[58] H. Chen, Y. Li, Y. Jiang, Y. Ma, and B. Vucetic. Distributed power splitting for SWIPT in relay interference channels using game theory. IEEE Transactions On Wireless Communications, 14(1):410-420, 2015, DOI: 10.1109/TWC.2014.2349892.

[59] D. Li, Y. Xu, X. Wang, and M. Guizani. Coalitional game theoretic approach for secondary spectrum access in cooperative cognitive radio networks. IEEE Trans. On Wireless Communications, 10(3):844-855, 2011, DOI: 10.1109/TWC.2011.011111.100216.

[60] I. Stupia, L. Sanguinetti, G. Bacci, and L. Vandendorpe. Power control in networks with heterogeneous users: A quasi-variational inequality approach. IEEE Trans. Signal Process., 63(21):5691-5705, Nov. 2015, DOI: 10.1109/TSP.2015.2452231.

[61] J. Wang, W. Guan, Y. Huang, R. Schober, and X. You. Distributed optimization of hierarchical small cell networks: A GNEP framework. IEEE J. Sel. Areas Commun., 35(2):249-264, Feb. 2017, DOI: $10.1109 /$ JSAC.2017.2658999.

[62] V. Pacifici and G. Dán. Convergence in player-specific graphical resource allocation games. IEEE J. Sel. Areas Commun., 30(11):2190-2199, Dec. 2012, DOI: 10.1109/JSAC.2012.121211.

[63] G. Scutari, D. P. Palomar, and S. Barbarossa. Competitive design of multiuser MIMO systems based on game theory: A unified view. IEEE Journal on Selected Areas in Communications, 26(7):1089-1103, Aug. 2008, DOI: 10.1109/JSAC.2008.080907.

[64] J. Wang, G. Scutari, and D. P. Palomar. Robust MIMO cognitive radio via game theory. IEEE Trans. on Signal Processing, 59(3):1183-1201, March 2011, DOI: 10.1109/TSP.2010.2092773.

[65] B. Fallah, B. X. Huang, and L. Tu. Distributed asynchronous game theoretic solutions for precoding strategies in multiuser MIMO systems. International Journal of Distributed and Parallel Systems (IJDPS), 3(4):133-143, July 2012, DOI: 10.5121/ijdps.2012.3414.

[66] P. Zhao, G. Fodor, G. Dan, and M. Telek. A game theoretic approach to setting the pilot-to-data power ratio in MU-MIMO systems. IEEE Transactions on Communications, 19(9):1604 - 1607, September 2017. DOI: 10.1109/TCOMM.2017.2778094.

[67] P. Zhao, G. Fodor, G. Dan, and M. Telek. A game theoretic approach to uplink pilot and data power control in multi-cell MU-MIMO systems. IEEE Transactions on Vehicular Technology, 19(9):1604 - 1607, June 2019, DOI: 10.1109/TVT.2019.2927127.

[68] S. Han, C. 1. I, Z. Xu, and C. Rowell. Large-scale antenna systems with hybrid analog and digital beamforming for millimeter wave 5G. IEEE Commun. Mag., 53(1):186-194, Jan. 2015, DOI: 10.1109/MCOM.2015.7010533.

[69] S. Rangan, T. S. Rappaport, and E. Erkip. Millimeter-wave cellular wireless networks: Potentials and challenges. Proc. IEEE, 102(3):366385, Mar. 2014, DOI: 10.1109/JPROC.2014.2299397.

[70] Z. Pi and F. Khan. An introduction to millimeter-wave mobile broadband systems. IEEE Communications Magazine, 49(6):101-107, 2011, DOI: 10.1109/MCOM.2011.5783993.10 
[71] S. Hur, T. Kim, D. J. Love, J. V. Krogmeier, T. A. Thomas, and A. Ghosh. Millimeter wave beamforming for wireless backhaul and access in small cell networks. IEEE Trans. Commun., 61(10):4391-4403, October 2013, DOI: 10.1109/TCOMM.2013.090513.120848.

[72] O. E. Ayach, S. Rajagopal, S. Abu-Surra, Z. Pi, and R. W. Heath. Spatially sparse precoding in millimeter wave MIMO systems. IEEE Trans. Wireless Commun., 13(3):1499-1513, March 2014, DOI: 10.1109/TWC.2014.011714.130846.

[73] A. Alkhateeb, O. El Ayach, G. Leus, and R. W. Heath. Channel estimation and hybrid precoding for millimeter wave cellular systems. IEEE J. Sel.Top. Signal Process., 8(5):831-846, October 2014 DOI: $10.1109 /$ JSTSP.2014.2334278.

[74] S. Kutty and D. Sen. Beamforming for millimeter wave communications: An inclusive survey. IEEE Communications Surveys Tutorials, 18(2):949973, September 2016, DOI: 10.1109/COMST.2015.2504600.

[75] S. Noh, M. D. Zoltowski, and D. J. Love. Training sequence design for feedback assisted hybrid beamforming in massive MIMO systems. IEEE Trans. Commun., 64(1):187-200, Jan. 2016, DOI: 10.1109/TCOMM.2015.2498184.

[76] J. Song, J. Choi, and D. J. Love. Common codebook millimeter wave beam design: Designing beams for both sounding and communication with uniform planar arrays. IEEE Trans. Commun., 65(4):1859-1872, Apr. 2017, DOI: 10.1109/TCOMM.2017.2665497.

[77] H. Shokri-Ghadikolaei, F. Boccardi, C. Fischione, G. Fodor, and M. Zorzi. Spectrum sharing in mmWave cellular networks via cell association, coordination, and beamforming. IEEE J.Sel.Areas Commun., 34(11):2902-2917, November 2016, DOI: 10.1109/JSAC.2016.2615259.

[78] M. Wu, D. Wuebben, A. Dekorsy, P. Baracca, V. Braun, and H. Halbauer. Hardware impairments in millimeter wave communications using OFDM and SC-FDE. In Proc. Smart Antennas (WSA), 2016 International ITG Workshop on, number ISBN:978-3-8007-4177-9, pages 1-8, March 2016.

[79] A. Khansefid, H. Minn, Q. Zhan, N. Al-Dhahir, H. Huang, and X. Du. Waveform parameter design and comparisons for millimeterwave massive MIMO systems with RF distortions. In Proc. IEEE Globecom Workshops (GC Wkshps), pages 1-6, Dec 2016, DOI: 10.1109/GLOCOMW.2016.7849081.

[80] H. Yan and D. Cabric. Digital predistortion for hybrid precoding architecture in millimeter-wave massive MIMO systems. In Proc. 42nd IEEE Int. Conf. on Acoustics, Speech and Signal Process., Mar. 2017, DOI: $10.1109 /$ ICASSP.2017.7952803

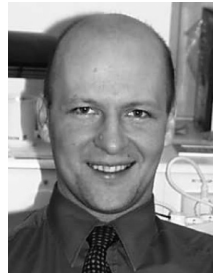

Gábor Fodor [SM'08] (Gabor.Fodor@ericsson.com) received his $\mathrm{Ph} . \mathrm{D}$. degree in electrical engineering from the Budapest University of Technology and Economics in 1998, his Docent degree from KTH Royal Institute of Technology, Stockholm, Sweden, in 2019, and his D.Sc. degree from the Hungarian Academy of Sciences in 2019. He is currently a master researcher with Ericsson Research and an adjunct professor with KTH Royal Institute of Technology. He was a co-recipient of the IEEE Communications Society Stephen O. Rice Prize in 2018. He is serving as the Chair of the IEEE Communications Society Full Duplex Emerging Technologies Initiative and as an Editor for IEEE Transactions on Wireless Communications.

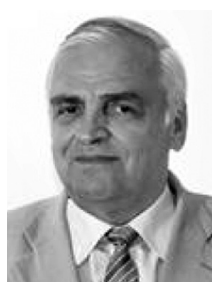

László Pap graduated from the Technical University of Budapest, Faculty of Electrical Engineering, Branch of Telecommunications. He became Dr. Univ. and Ph.D. in 1980, and Doctor of Sciences in 1992. In 2001 and 2007 he has been elected as a Correspondent and Full Member of the Hungarian Academy of Sciences. His main fields of the research are the electronic systems, nonlinear circuits, synchronization systems, modulation and coding, spread spectrum systems, CDMA, multiuser detection and mobile communication systems. His main education activity has covered the fields of electronics, modern modulation and coding systems, communication theory, introduction to mobile communication. Professor Pap had been Head of the Dept. of Telecommunications, the Dean of the Faculty of Electrical Engineering at Budapest University of Technology and Economics, and Vice Rector of the University.

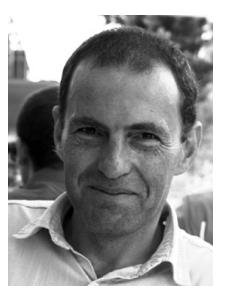

Miklós Telek graduated as an electrical engineer at the Faculty of Electrical Engineering, Technical University of Budapest in 1987. He received the candidate of science and the MTA doctor degree from the Hungarian Academy of Sciences in 1995 and 2004, respectively. Since 1990 he has been with the Technical University of Budapest. Currently, he is a professor at Department of Networked Systems and Services. Since 2012, he is heading the MTA-BME Information Systems Research Group of the Hungarian Academy of Sciences. His current research interests include various aspects of stochastic performance modeling and analysis of computer and communication systems. 